\title{
Numerical Simulation of Low-Permeability Reservoirs with considering the Dynamic Boundary Layer Effect
}

\author{
Chuanzhi Cui, ${ }^{1}$ Yingfei Sui $\mathbb{D}^{1},{ }^{1}$ Xiangzhi Cheng, ${ }^{2}$ Yinzhu Ye$^{2}$ and Zhen Wang ${ }^{1}$ \\ ${ }^{1}$ College of Petroleum Engineering, China University of Petroleum, Qingdao 266580, China \\ ${ }^{2}$ Petrochina Research Institute of Petroleum Exploration \& Development, Department of Logging \& Remote Sensing Technology, \\ Beijing 100083, China
}

Correspondence should be addressed to Yingfei Sui; 1240321277@qq.com

Received 5 October 2020; Revised 28 December 2020; Accepted 10 March 2021; Published 22 March 2021

Academic Editor: Yu Wang

Copyright (c) 2021 Chuanzhi Cui et al. This is an open access article distributed under the Creative Commons Attribution License, which permits unrestricted use, distribution, and reproduction in any medium, provided the original work is properly cited.

Surface active components, salt component, and polar molecules in the fluid may adsorb on the solid surface and form the boundary layer during low-speed flow in a porous medium, which will influence the flowing law in the porous medium. Previous studies on flowing in low-permeability reservoirs mainly focus on the effects of the threshold pressure gradient. But few of them have considered the time-varying effect of the boundary layer thickness in solving the numerical simulation. The correlation among the boundary layer thickness and pressure gradient was established by regressing the experimental data of boundary thickness versus pressure. On this basis, the mathematical model of oil-water two-phase flow which involves influence of the boundary layer was constructed, and the comparative analysis of the development effect is performed. Results demonstrated that the boundary layer thickness is sensitive to the throat radius and pressure gradient, and the boundary layer thickness decreases dynamically with the increase of pressure gradient. The displacement velocity and accumulative oil production with boundary layer effect decrease when comparing with that without the boundary layer effect. Meanwhile, the boundary layer accelerates the breakthrough of water. With the reduction of production pressure difference, the difference between accumulative oil production with and without the boundary layer effect increases, which indicate that the dynamic effect of the boundary layer is intensified.

\section{Introduction}

Due to the interface interaction between solid and liquid, there is a fluid layer on the surface of the porous media which fluid properties change with the flow and the fluid in it is called the boundary layer fluid. The formation of the boundary layer on the solid phase surface is related with the interaction force between the solid surface and molecules. This is attributed to two reasons: the induction effect of the solid phase field close to the adsorption layer and the effects of the molecular field in the adsorption layer $[1,2]$. Rock particles have a stronger attraction to fluid molecules closer to the pore channel surface and fluid molecules on the solid surface arrange more tightly. Since the boundary layer fluid is the closest to the solid phase surface, the interaction between rock molecular and fluid molecules reaches the maximum and the fluid molecules are in an ordered arrangement, showing the characteristics of solids [3] (Figure 1).

Recently, researches on the boundary layer have attracted wide attentions. Huang et al. [4] studied the selectivity of fluid through low-permeability porous media, elaborated the properties of fluid, and proposed the new nonlinear flowing equation. Yang et al. [5] carried out a microtubule experiment by using the independently developed equipment and studied the flowing laws under the microscale. Flows in the microtube were calculated by the dissipative particle dynamics method, and the microscale flow effect was simulated at present [6-9]. At the microlevel, particle dynamics is used to simulate and characterize the influence of the boundary layer on flowing 


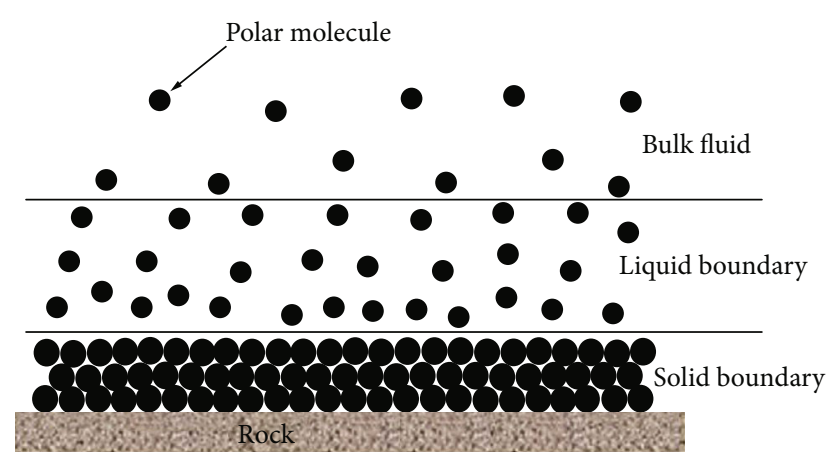

Figure 1: Boundary layer structure.

characteristics, which has become the hotspot to study the flowing law of the reservoir $[10,11]$. Wang and Sheng [12, 13] analyzed the flow mechanism, considered the boundary effect, and illustrated that the low-speed non-Darcy flow pattern was composed of a nonlinear flow part and linear flow part. Zhongwei et al. [14, 15] used the threshold pressure gradient and permeability modulus to characterize the low-speed non-Darcy flow and stress sensitivity, respectively, considering the boundary effect, and established a multilinear dynamic pressure model of multistage fractured horizontal wells considering low-speed nonDarcy flow and stress sensitivity. Tian et al. [16] modified the Purcell solution based on the collected experimental results and proposed an improved solution considering the influence of boundary layer, Jamin, and wettability.

There are few numerical simulation methods that directly consider the dynamic change of the boundary layer. By combining the study experiment on the microscale flow in this paper, the influencing factors of the boundary layer were analyzed and the quantitative characterization method of dynamic changes of the boundary layer was established. And contrast analyses of the development effects with considering the boundary layer were carried out.

\section{Characterization Methods of Dynamic Features of the Boundary Layer Thickness}

The pore space in the rocks was viewed as unidiameter capillary tubes. Flowing characteristics of fluids in the reservoir were studied by using the capillary tube model $[17,18]$. The effective capillary tube radius under the same conditions was calculated by the Poiseuille formula, thus calculating the thickness of the boundary layer.

For one capillary tube, the actual radius and length were $r_{0}$ and $L$. In the existence of pressure difference $(\Delta p)$, the fluid only flows in the effective radius range and the flow passing through the capillary tube is $Q$. It can be taken from the Poiseuille formula that

$$
Q=\frac{\pi r_{E}^{4} \Delta p}{8 \mu L} .
$$

It can be known from Equation (1) that the effective radius is

$$
r_{E}=\sqrt[4]{\frac{8 \mu L Q}{\pi \Delta p}} .
$$

The boundary layer thickness is equal to the difference between the actual radius and effective radius of the capillary tube:

$$
\delta=r_{0}-r_{E} .
$$

After the dimensionless processing, the dimensionless thickness of the boundary layer could be gained:

$$
\delta^{*}=\frac{\delta}{r_{0}} .
$$

It can be known from Equations (2)-(4) that the boundary layer thickness is related to the capillary tube radius, pressure gradient, and fluid viscosity. The boundary layer has the characteristics of dynamic changes, which are attributed to the fluctuation of the pressure gradient in the development process. Yang et al. [5] disclosed the relationship between the dimensionless boundary layer thickness and pressure gradient under different capillary tube radii through the microscale capillary flow experiment (Figure 2).

In Figure 2, the dimensionless boundary layer thickness of the fluid is negatively correlated with the pressure gradient. With the reduction of capillary radius, the dimensionless boundary layer thickness increases.

The pressure gradient and dimensionless boundary layer thickness were drawn in the semilogarithm coordinates (Figure 3). The curve can be divided into two sections. The fast descending section is the movable part in the boundary layer, and the section which is approximately horizontal can be viewed as the solidification layer in the boundary layer. When calculating the dynamic change of boundary layer thickness, the horizontal line segment is usually not considered, so the dimensionless thickness of boundary layer has a linear relationship with the logarithm of pressure gradient [19-21]. It is found that the dimensionless thickness of the boundary layer has an exponential relationship with the capillary radius and a linear relationship with the fluid viscosity [16]. Therefore, the dimensionless thickness of the boundary layer can be expressed by Equation (5) [16].

$$
\delta^{*}=a \cdot e^{b r}(\nabla p)^{c} \cdot \mu .
$$

In Equation (5), $a, b$, and $c$ are the fitting coefficients. Using the data of the fast descending section in Figure 3, multiple regression fitting is carried out. In the experimental data shown in Figure 3, the fluid viscosity is a constant value of $1 \mathrm{MPa} \cdot \mathrm{s}$. The fitting results are shown in Table 1 and Figure 4. 


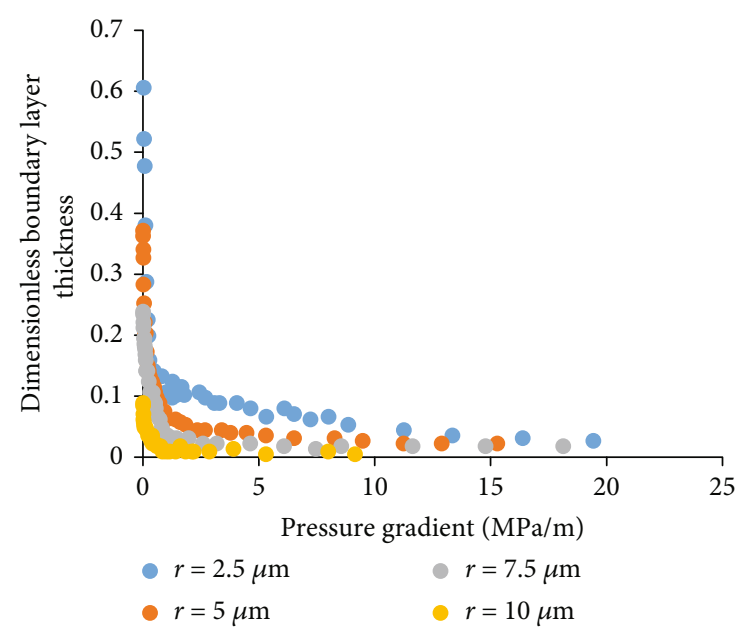

FIGURE 2: The relationship between dimensionless boundary layer thickness and pressure gradient under different capillary tube radii [5].

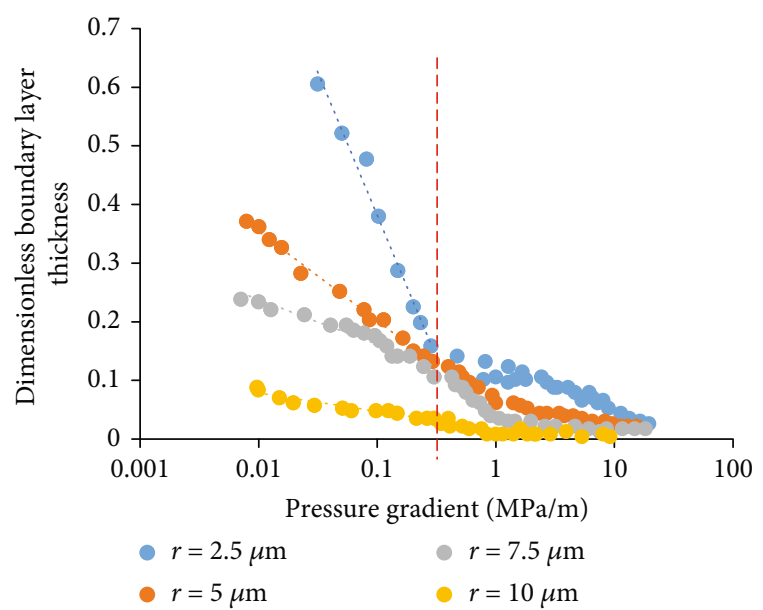

FIGURE 3: The relationship between the pressure gradient and dimensionless boundary layer thickness in the semilogarithmic coordinates.

\section{Reservoir Numerical Simulation with considering Dynamic Characteristics of the Boundary Layer}

3.1. Relationship between the Boundary Layer Thickness and Permeability. Under the condition that the real rock and the imaginary rock have the same external dimension, the same fluid property, the same acting pressure difference, and the rock surface is oil wet, according to the same flow rate, the combination of the Poiseuille formula and Darcy formula can be obtained:

$$
\begin{aligned}
& Q=N \frac{\pi r_{E}^{4} \Delta p}{8 \mu L \tau}=\frac{K A \Delta p}{\mu L}, \\
& K=n \cdot \frac{\pi r_{E}^{4}}{8 \tau} .
\end{aligned}
$$

TABle 1: Fitting coefficient of boundary layer thickness characterization formula.

\begin{tabular}{lcc}
\hline Fitting coefficient & Fitting value & Goodness of fit, $R$ \\
\hline$a$ & 0.3371 & \\
$b$ & -0.2371 & 0.975 \\
$c$ & -0.2938 & \\
\hline
\end{tabular}

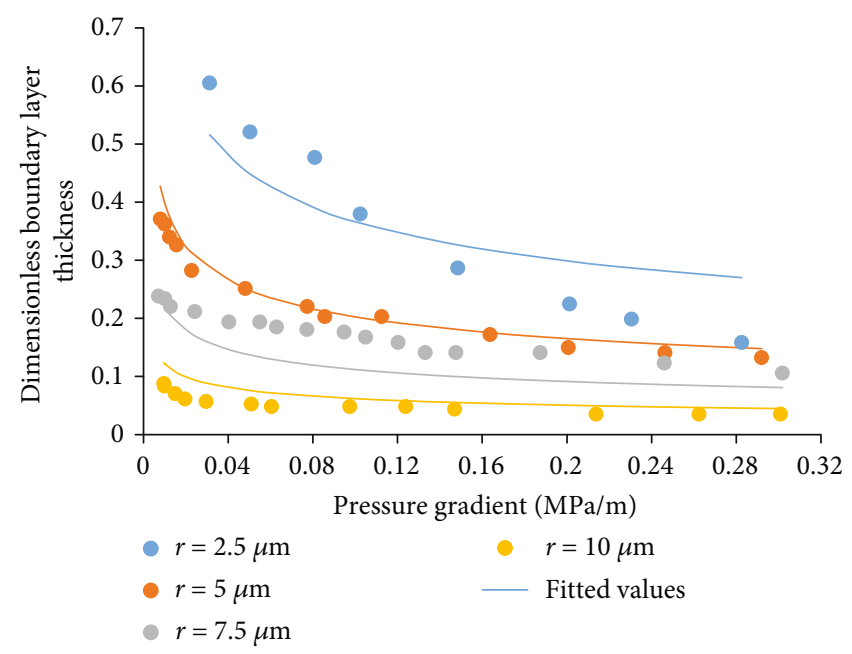

FIGURE 4: Fitting curve of boundary layer thickness under different capillary radii.

When the actual radius of the capillary tube is $r_{0}$ and the boundary layer thickness is $\delta$, the permeability is

$$
K=K_{0} \cdot \frac{\left(r_{0}-\delta\right)^{4}}{r_{0}^{4}}
$$

where $Q$ is the flow volume of fluid $\left(\mathrm{m}^{3} / \mathrm{s}\right), K_{0}$ is the permeability without considering the boundary layer $\left(\mu \mathrm{m}^{2}\right), K$ is the permeability with considering the boundary layer $\left(\mu \mathrm{m}^{2}\right), n$ is the number of capillary tubes on the unit area $(n=N / A), \tau$ is the tortuosity, $\delta$ is the boundary layer thickness $(\mu \mathrm{m}), r_{E}$ is the effective radius of capillary tube $(\mu \mathrm{m})$, and $r_{0}$ is the actual radius of capillary tube $(\mu \mathrm{m})$.

3.2. Percolation Model with considering the Dynamic Characteristics of the Boundary Layer. The equations of continuity of oil and water phases under the one-dimensional unidirectional flow are

$$
\begin{gathered}
-\frac{\partial v_{w}}{\partial x}+q_{v w}=\varphi(x, t) \frac{\partial S_{w}}{\partial t}, \\
-\frac{\partial v_{o}}{\partial x}+q_{v o}=\varphi(x, t) \frac{\partial S_{o}}{\partial t} .
\end{gathered}
$$




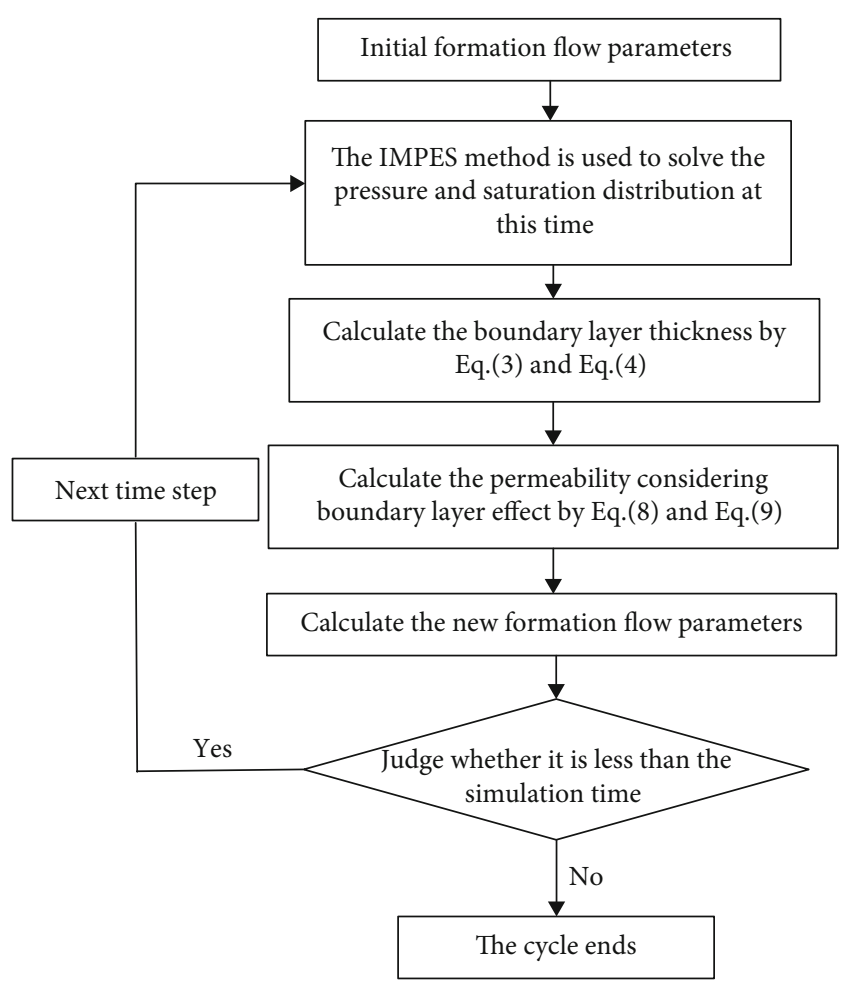

Figure 5: Flow chart of percolation model considering dynamic characteristics of the boundary layer.

The kinematic equation is

$$
\begin{gathered}
v_{w}=-\frac{K_{0}\left(r_{0}-\delta\right)^{4} K_{r w}}{r_{0}{ }^{4} \mu_{w}} \frac{\partial p}{\partial x}, \\
v_{o}=-\frac{K_{0}\left(r_{0}-\delta\right)^{4} K_{r o}}{r_{0}^{4} \mu_{o}} \frac{\partial p}{\partial x} .
\end{gathered}
$$

The auxiliary equation is $S_{w}+S_{o}=1$.

In initial conditions, the initial pressure is the initial pressure of the reservoir, and the initial water saturation is the irreducible water saturation. The formula is as follows:

$$
\left.P\right|_{t=0}=P_{i} ;\left.S_{w}\right|_{t=0}=S_{w c}
$$

In boundary conditions, the flow rate of the water phase at the inlet end is the water injection volume, and the flow rate of the liquid phase at the outlet end is the water injection volume. The formula is as follows:

$$
\left.q_{v w}\right|_{x=0}=q_{v} ;\left.q_{v}\right|_{x=L}=-q_{v} .
$$

In the above equations, $p, S, \mu, q, K, K_{r}$, and $\phi$ are pressure, saturation, viscosity, volume flow rate, absolute permeability, relative permeability, and porosity, respectively. The subscripts $o, w$ represent oil phase and water phase.

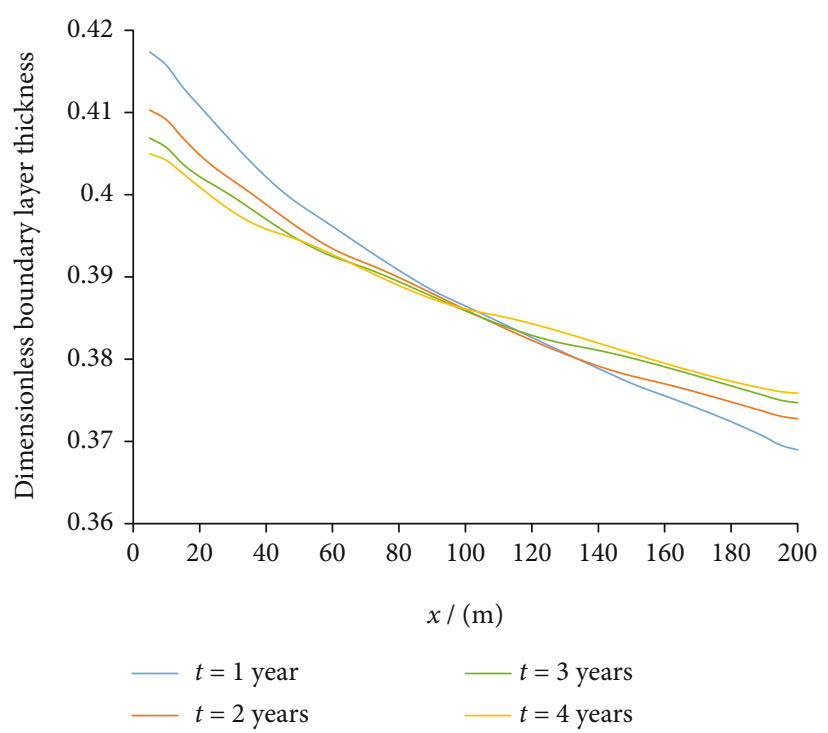

FIGURE 6: Relation curves between the dimensionless boundary layer thickness and position at different times.

The kinematic equation is brought into the equation of continuity and the water saturation is eliminated:

$$
\lambda \frac{\partial P}{\partial x}+q_{v}=0
$$

where

$$
\lambda=\lambda_{w}+\lambda_{o}=\frac{K_{0}\left(r_{0}-\delta\right)^{4} K_{r w}}{r_{0}^{4} \mu_{w}}+\frac{K_{0}\left(r_{0}-\delta\right)^{4} K_{r o}}{r_{0}^{4} \mu_{o}} .
$$

Equation (13) was discretized, and the pressure was solved by the chasing method [22]. The equation which only involves the water saturation could be gained by bringing the calculated pressure into the equation of continuity of water phase, and then, the water saturation is solved. The solution procedure is as follows: (1) According to the initial permeability and fluid viscosity, the distribution of pressure and saturability at $i$ was disclosed by IMPES method [23, 24].(2) Calculate the boundary layer thickness from Equations (3) and (4). (3) The permeability considering boundary layer effect is calculated by Equations (7) and (8), and the diversion coefficient is updated. The formation flow parameters after permeability update are calculated [25]. (4) Judge whether it is less than the simulation time. If yes, it returns to the parameters at the time of calculation in step (1). Otherwise, the cycle ends. The program calculation flow is shown in Figure 5.

\section{Model Solving and Result Analysis}

The percolation model was solved with considering the effects of the boundary layer on reservoir permeability. The distance between the injection well and production well was set $200 \mathrm{~m}$. Effective thickness was set $10 \mathrm{~m}$. And the initial 


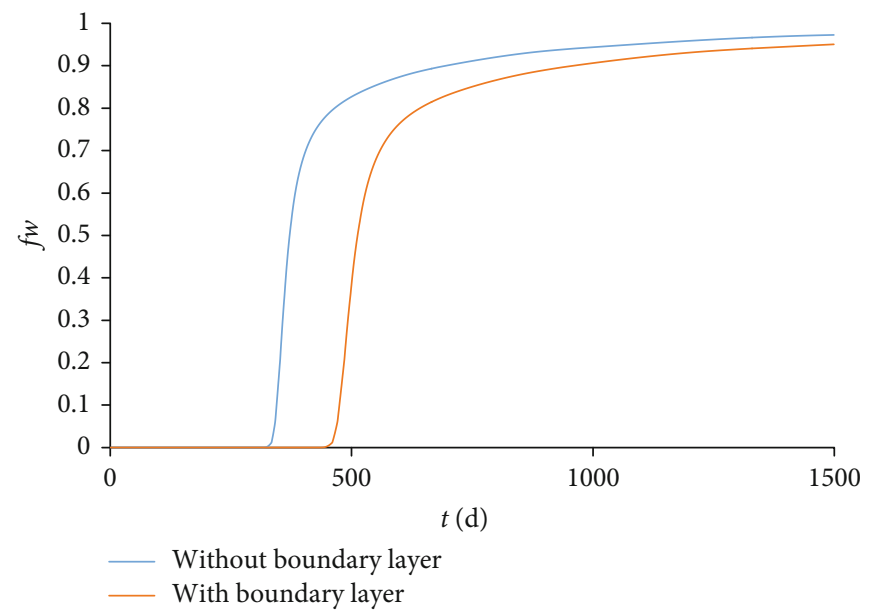

FIGURE 7: Comparison of water content distribution with and without considering the boundary layer.

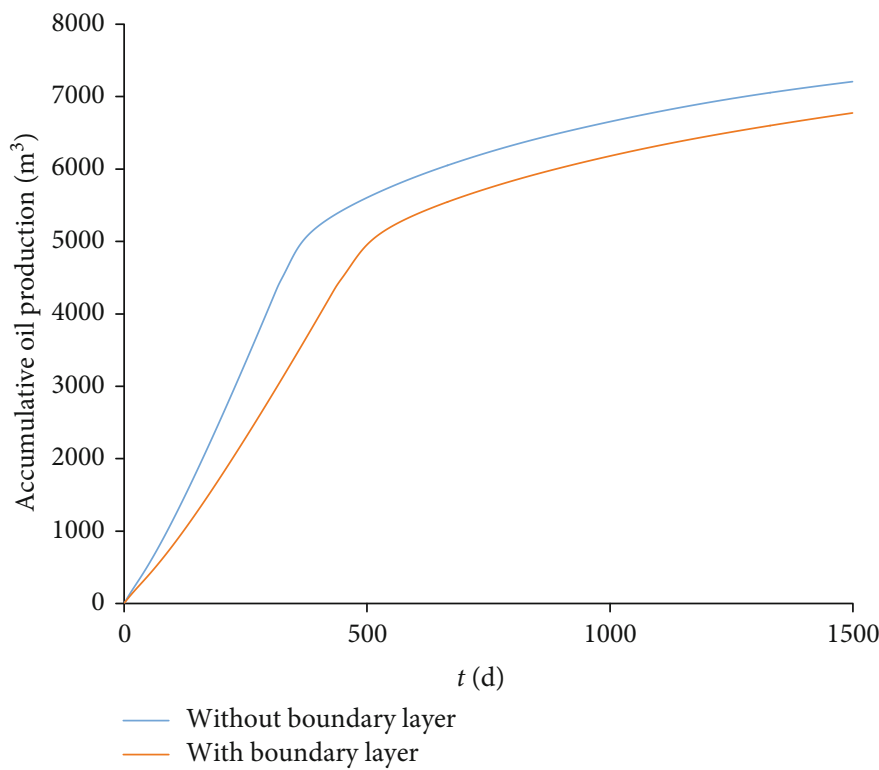

FIGURE 8: Comparison of accumulative oil production with and without considering the boundary layer.

permeability of the reservoir was set $10 \times 10^{-3} \mu \mathrm{m}^{2}$. The rest of parameters include porosity (0.26), water viscosity $(0.5 \mathrm{MPa} \cdot \mathrm{s})$, oil viscosity $(8 \mathrm{MPa} \cdot \mathrm{s})$, oil $\left(850 \mathrm{~kg} / \mathrm{m}^{3}\right)$, water density $\left(1000 \mathrm{~kg} / \mathrm{m}^{3}\right)$, and radius of pore channel in rocks $(2.5 \mu \mathrm{m})$. Production under fixed pressure difference was adopted, and the pressure difference was $10 \mathrm{MPa}$. The time of simulation production was 1500 days. The variation law of the boundary layer thickness at different times was drawn. The change of oil production and water content with and without considering the boundary layer thickness were compared.

It can be seen from Figure 6 that the boundary layer thickness decreases gradually from the injection well to the production well. The flowing resistance is positively related with the distance between the injection well and the production well caused by the higher oil viscosity than water viscosity. Therefore, the pressure difference from the injection well to the production well increases gradually, thus resulting in the gradual reduction of the boundary layer thickness.

The boundary layer thickness becomes increasingly stable as time goes on at the same pressure difference. This is because sweep length of the water phase increase gradually until water breakthrough as development continues. Accordingly, the change of flowing resistance between injection and production wells gradually slows down, and the change of pressure tends to be stable, thus decreasing changes of the boundary layer thickness gradually.

Comparisons of water content and accumulative oil production with and without the boundary layer effect are shown in Figures 7 and 8 . When the boundary layer effect is considered, the displacement speed becomes slower leading to the obvious delay of water breakthrough comparing with that without boundary layer effect at the same pressure difference. This is because the boundary layer decreases the 


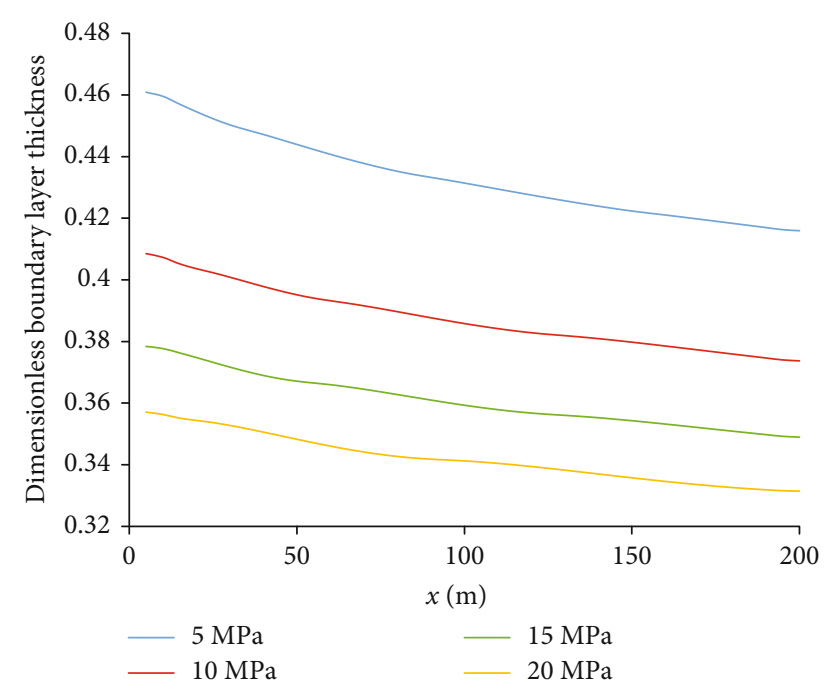

FiguRE 9: Relationship between the dimensionless boundary layer thickness and location under different injection production pressures.

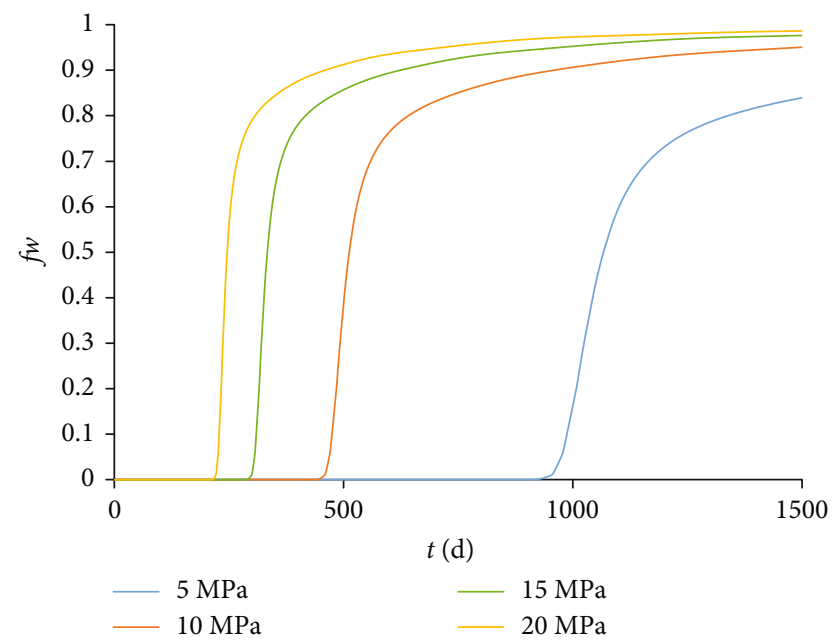

Figure 10: Variation curve of water content under different pressure gradients with considering the boundary layer.

absolute permeability of the reservoir and increases the flowing resistance. In addition, when considering the effect of boundary layer, the daily liquid production and oil production decrease obviously owing to the decrease of permeability. It can be seen from Figure 8 that the boundary layer effect leads to a significant decrease in cumulative oil production. Because of the prominent boundary layer effect in lowpermeability reservoir, it is more close to the actual development situation to consider the influence of boundary layer in the study of flow law and the prediction of development effect.

In order to discuss the influence of the boundary layer on the development effect under different pressure gradients, the models with pressure difference of $5 \mathrm{MPa}, 10 \mathrm{MPa}, 15 \mathrm{MPa}$, and $20 \mathrm{MPa}$ were calculated, respectively.

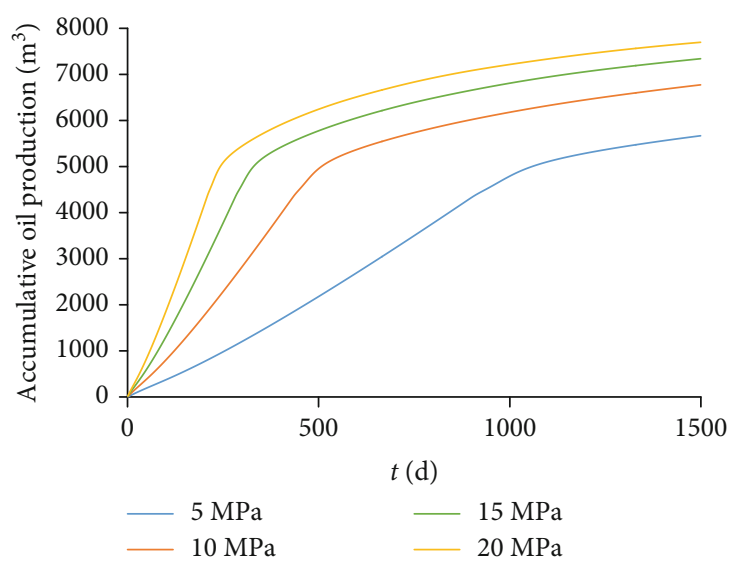

FIGURE 11: Variation curve of accumulative oil production under different pressure gradients with considering the boundary layer.

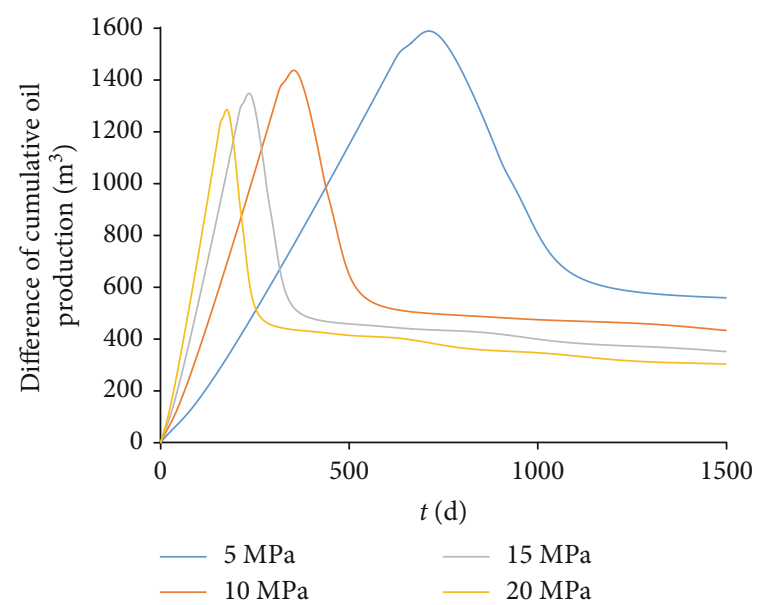

FIGURE 12: Difference of accumulative oil production with and without considering the boundary layer.

Figure 9 shows the relationship curve between the thickness of the boundary layer and the position under different pressure gradients. It can be seen that the boundary layer thickness gradually decreases with the increase of pressure difference at the same position and the rate of decrease becomes slower. This is because that the increase of pressure difference leads to the increase of displacement pressure gradient and the decrease of boundary layer thickness in the case of the same injection production well spacing.

The variation curves of water content and the accumulative oil production under different pressure differences are shown in Figures 10 and 11. Due to the influence of pressure gradient on the boundary layer thickness, the permeability of reservoir is smaller where the pressure gradient is smaller. Therefore, the displacement velocity becomes slower and the accumulative oil production decreases with the reduction of pressure difference. In addition, with the decrease of pressure difference, the effect of the boundary layer is more and more significant, and the influence of boundary layer on water content and cumulative oil production increases gradually. 
The difference of accumulative oil production with and without considering the boundary layer is shown in Figure 12. The difference of cumulative oil production increases almost linearly before water breakthrough, and the difference of cumulative oil production increases more slowly when the injection production pressure difference is small. This is because the change of water cut and pressure gradient in the reservoir is very little in the later stage of development, which leads to the small change of boundary layer thickness. In the late stage of development, the difference of cumulative oil production increases with the decrease of injection production pressure difference. Therefore, the boundary layer has an adverse impact on the development effect, and the adverse impact increases with the decrease of pressure difference.

\section{Conclusions}

(1) There are prominent boundary layer effects during the exploitation of low-permeability oil reservoirs. The boundary layer thickness is related with the radius of the pore throat, pressure gradient, and fluid viscosity

(2) Due to the change of pressure gradient, the boundary layer is characterized by dynamic change, and the thickness of boundary layer decreases with the increase of pressure gradient. The existence of boundary layer results in the decrease of reservoir permeability and the poor development effect

(3) The oil-water percolation model with considering the boundary layer thickness demonstrates that the displacement is slow when the boundary layer is considered, accompanied with delayed water breakthrough and reduced accumulative oil production. Along with the continuous water flooding development, the change of boundary layer thickness gradually slows down

(4) With the reduction of pressure difference, the influence of the boundary layer on water content and cumulative oil production increases gradually. Meanwhile, the boundary layer has an adverse impact on the development effect, and the adverse impact increases with the decrease of pressure difference

\section{Data Availability}

The [DATA TYPE] data used to support the findings of this study are included within the article.

\section{Conflicts of Interest}

The authors declare that they have no conflicts of interest.

\section{Acknowledgments}

This work was supported by the National Natural Science Foundation of China (51974343) and Shandong Province Natural Science Foundation (ZR2017MEE054). The first author would like to acknowledge the College of Petroleum Engineering at China University of Petroleum (Qingdao) for providing experimental equipment.

\section{References}

[1] Y. J. Gong, S. B. Liu, L. Jiang, X. J. Yuan, and S. Z. Tao, "Experiment study of seepage characteristic and mechanism in tight gas sands: a case from Xujiahe reservoir of Sichuan Basin," Natural Gas Geoscience, vol. 25, no. 6, pp. 804-808, 2014.

[2] J. Chengchao, Z. Caineng, L. Jianzhong, L. Denghua, and Z. Min, "Assessment criteria, main types, basic features and resource prospects of the tight oil in China," Acta Petrolei Cinica, vol. 33, no. 3, pp. 343-350, 2012.

[3] L. Weizhong, M. Zhangzhen, and S. Yongchen, "Modeling on non-Darcy phenomena of gas flow in tight porous media using lattice Boltzmann method," Journal of Liaoning Technical University (Natural Science), vol. 29, no. 3, pp. 412-415, 2010.

[4] Y. Huang, Z. Yang, Y. He, X. Wang, and Y. Luo, "Nonlinear porous flow in low permeability porous media," Mechanics in engineering, vol. 35, no. 5, pp. 1-7, 2013.

[5] L. Yang, L. Qun, L. Xiangui, and X. Hanmin, "Characteristics of micro scale nonlinear filtration," Petroleum Exploration and Development, vol. 38, no. 3, pp. 336-340, 2011.

[6] X. Tian, L. Cheng, R. Cao, N. An, M. Zhang, and Y. Wang, "Characteristics of boundary layer in micro and nano throats of tight sandstone oil reservoirs," Chinese journal of computation physics, vol. 33, no. 6, pp. 713-724, 2016.

[7] X. Fan, N. Phan-Thien, N. T. Yong, X. Wu, and D. Xu, "Microchannel flow of a macromolecular suspension," Physics of Fluids, vol. 15, no. 1, pp. 11-21, 2003.

[8] A. Kumar, Y. Asako, E. Abu-Nada, M. Krafczyk, and M. Faghri, "From dissipative particle dynamics scales to physical scales: a coarse-graining study for water flow in microchannel," Microfluidics and Nanofluidics, vol. 7, no. 4, pp. 467-477, 2009.

[9] J. Kikan and O. A. J. R. Pestra, "Perturbation analysis of stresssensitive reservoirs (includes associated papers 25281 and 25292)," Society of Petroleum Engineers Formation Evaluation, vol. 6, no. 3, pp. 379-386, 1991.

[10] R. K. Zhu, S. T. Wu, L. Su, J. W. Cui, Z. G. Mao, and X. X. Zhang, "Problems and future works of porous texture characterization of tight reservoirs in China," Acta Petrolei Sinica, vol. 37, no. 11, pp. 1323-1334, 2016.

[11] X. Wang, X. Hou, M. Hao, and T. Yang, "Pressure transient analysis in low-permeable media with threshold gradients," Acta Petrolei Sinica, vol. 32, no. 5, pp. 847-851, 2011.

[12] X. Wang and J. J. Sheng, "Effect of low-velocity non-Darcy flow on well production performance in shale and tight oil reservoirs," Fuel, vol. 190, pp. 41-46, 2017.

[13] X. Wang and J. J. Sheng, "A self-similar analytical solution of spontaneous and forced imbibition in porous media," Advances in Geo-Energy Research, vol. 2, no. 3, pp. 260-268, 2018.

[14] Z. Wu, C. Cui, G. Lv, S. Bing, and G. Cao, “A multi-linear transient pressure model for multistage fractured horizontal well in tight oil reservoirs with considering threshold pressure gradient and stress sensitivity," Journal of Petroleum Science and Engineering, vol. 172, pp. 839-854, 2018.

[15] Z. Wu, L. Dong, C. Cui, X. Cheng, and Z. Wang, “A numerical model for fractured horizontal well and production 
characteristics: comprehensive consideration of the fracturing fluid injection and flowback," Journal of Petroleum Science and Engineering, vol. 187, p. 106765, 2020.

[16] X. Tian, L. Cheng, Y. Yan, H. Liu, W. Zhao, and Q. Guo, “An improved solution to estimate relative permeability in tight oil reservoirs," Journal of Petroleum Exploration \& Production Technology, vol. 5, no. 3, pp. 305-314, 2015.

[17] H. Li, H. K. Guo, and H. Li, "Thickness analysis of bound water film in tight reservoir," Natural Gas Geoscience, vol. 26, no. 1, pp. 186-191, 2015.

[18] L. Aifen, Reservoir Physics, University of Petroleum Press, Dongying, 2011.

[19] H. Ling, Z. Yang, Q. Xiao, and Q. Xu, "Study 8on a new porous flow model in tight oil formation," Science Technology and Engineering, vol. 13, no. 26, pp. 7624-7627, 2013.

[20] P. Dong, G. Lei, B. Y. Ji, and S. B. Tian, "Nonlinear seepage regularity of tight sandstone reservoir with consideration of medium deformation," Chinese Journal of Rock Mechanics and Engineering, vol. 32, no. 2, pp. 3187-3195, 2013.

[21] X. Yunting, X. Qi, G. Yonggui, and Y. Zhengming, Study and Application of Percolation Mechanism in Low Permeability Reservoir, Petroleum Industry Press, Beijing, 2006.

[22] L. Weiguo and T. Dengke, Numerical Method, University of Petroleum Press, Dongying, 2009.

[23] Y. Huaijian, Z. Weiyao, Z. Mingjiang, Z. H. Meng, and S. O. Hong-qing, "Analysis of formation pressure distribution and productivity of low permeability tight gas reservoirs with low velocity non-Darcy flow," Special oil \& gas reservoirs, vol. 19, no. 1, pp. 70-73, 2012.

[24] L. Shuxia and G. Jianwei, Reservoir Numerical Simulation, University of Petroleum Press, Dongying, 2009.

[25] Z. Jianguo, Reservoir Seepage Mechanics, vol. 4, University of Petroleum Press, Dongying, 2009. 